\title{
Diagnostik des chronischen Unterbauchschmerzes
}

\author{
M. Nagel U. Wehrmann $\quad$ B. Ringelband \\ Klinik und Poliklinik für Viszeral-, Thorax- und Gefäßchirurgie, Universitätsklinikum Carl Gustav Carus, Technische Universität Dresden
}

\author{
Schlüsselwörter \\ Unterbauchschmerz · Laparoskopie · Laparoskopische Appen- \\ dektomie
}

\section{Zusammenfassung}

Der chronische Unterbauchschmerz stellt den behandelnden Arzt vor erhebliche differentialdiagnostische Probleme. Die vorgestellte Studie soll den Wert der Laparoskopie im diagnostischen Konzept aufzeigen. Dazu führten wir zwischen Oktober 1993 und Juni 1998 bei 100 Patienten mit der klinischen Diagnose "chronischer Unterbauchschmerz" eine Laparoskopie durch. Bei 14 Patienten ließ sich ein morphologisches Korrelat unabhängig von der Appendix finden. 86 Patienten wurden appendektomiert, wobei die histologische Untersuchung des Präparats in 82 Fällen (95,3\%) einen pathologischen Befund aufwies. 80 Patienten (93\%) blieben auch während der Nachuntersuchungen beschwerdefrei. Die Laparoskopie stellt ein sicheres Verfahren zur Diagnostik und Behandlung chronischer Unterbauchschmerzen dar. Bei fehlendem Korrelat für die Beschwerden sollte in gleicher Sitzung die laparoskopische Appendektomie durchgeführt werden.

\section{Einleitung}

Chronisch persistierende oder chronisch rezidivierende Unterbauchschmerzen stellen im klinischen Alltag ein nicht so seltenes Problem dar. Die Patienten berichten oft über eine Anamnese von Monaten oder Jahren, und in die Diagnostik waren häufig bereits zahlreiche medizinische Disziplinen involviert. Trotz Ausnutzung moderner diagnostischer Möglichkeiten läßt sich zur Frustration von Patienten und Arzt jedoch häufig keine klare Diagnose stellen, die die Beschwerden erklärt. Im folgenden wird ein diagnostisches Konzept aufgezeigt, welches zur Klärung des schwer faßbaren Krankheitsbilds des chronischen Unterbauchschmerzes rasch und mit hoher Zuverlässigkeit beitragen kann.

$\begin{array}{ll}\text { KARGER } & \text { @ 2000 S. Karger GmbH, Freiburg } \\ \begin{array}{l}\text { Fax }+497614520714 \\ \begin{array}{l}\text { E-mail Information@Karger.de } \\ \text { www.karger.com }\end{array}\end{array} & \begin{array}{l}\text { Accessible online at: } \\ \text { www.karger.com/journals/cga }\end{array}\end{array}$

\author{
Key Words \\ Lower abdominal pain · Laparoscopy · Laparoscopic appen- \\ dectomy
}

\section{Summary}

The differential diagnosis chronic lower abdominal pain can be problematic, and this symptom may lead to several diagnostic procedures. The purpose of this study was to evaluate the usefulness of laparoscopy in the diagnostic concept. From October 1993 to June 1998 we performed 100 laparoscopies in patients with chronic or recurrent lower abdominal pain. In 14 patients we found a substrate for the reported pain, which was independent of the appendix. In 86 patients we performed an appendectomy. In 62 of the specimens (95.3\%) the histological study showed pathological findings. 80 patients (93\%) reported no further complaints during follow-up. Laparoscopy is a safe procedure for diagnostics and treatment of patients with chronic lower abdominal pain. If no other explanation for the symptoms is found, laparoscopic appendectomy should be performed.

\section{Differentialdiagnose}

Schmerzen im Unterbauch können durch eine Vielzahl verschiedener Krankheiten hervorgerufen werden. Im Vordergrund stehen die entzündlichen Erkrankungen, die je nach befallenem Organsystem dem chirurgischen, gynäkologischen oder urologischen Fachgebiet zugeordnet werden können (Tab. 1).

Bei akutem Verlauf der entzündlichen Erkrankung führt die klinische Symptomatik meist rasch zur (in der Regel operativen) Klärung des Problems. Chronischer oder in Intervallen rezidivierender Schmerz zieht hingegen häufig eine langwierige und oft ineffektive Diagnostik nach sich. Neben entzündlichen Affektionen des weiblichen Genitales sind chronisch entzündliche Darmerkrankungen

PD Dr. M. Nagel

Klinik und Poliklinik für Viszeral-, Thorax- und Gefäßchirurgie

Universitätsklinikum Carl Gustav Carus

Technische Universität Dresden

Fetscherstraße 74, D-01307 Dresden

Tel. + 49 3514 58-2863, Fax -4317 
Tab. 1. Differentialdiagnose chronisch persistierender oder chronisch rezidivierender Unterbauchschmerzen

\begin{tabular}{ll}
\hline Chirurgisch & Gynäkologisch \\
Chronische Appendizitis & Adnexitis, Salpingitis \\
M. Crohn, Colitis ulcerosa & Endometriose \\
Adhäsionen & Ovarialzyste \\
Hernien & \\
Urologisch & Sonstige \\
Zystitis & Yersiniose \\
Urolithiasis & Shigellen \\
Pyelonephritis & Ogilvie-Syndrom \\
& Koprostase \\
\hline
\end{tabular}

(Morbus Crohn, Colitis ulcerosa) oder eine Divertikulitis zu erwägen. Die Existenz einer chronischen oder einer chronisch rezidivierenden Appendizitis wird in der Literatur kontrovers diskutiert, wobei jedoch histopathologische Befunde für das Vorliegen einer solchen Entität sprechen.

Unter den nichtentzündlichen Ursachen sind vor allem peritoneale Adhäsionen nach vorausgegangenen Operationen, aber auch nach Affektionen des weiblichen Genitalsystems zu erwägen. Das Beschwerdebild ist dabei meist nur gering bis mäßig ausgeprägt. Der chronische Verlauf führt bei den betroffenen Patienten nicht selten zur Beeinflussung der psychischen Konstitution, was die Beurteilung der Symptomatik im klinischen Alltag nicht erleichtert.

Je nach Lebensalter müssen weitere Ursachen in die Differentialdiagnose einbezogen werden. Bei Kindern werden Beschwerden anderer Lokalisationen häufig auf den Bauchraum projiziert. Des weiteren führen systemische Infekte oft zu einer begleitenden Lymphadenitis der Lymphknoten des Mesenteriums. An infektiologischen Ursachen muß aber auch in anderen Altersstufen und vor allem bei immunsupprimierten oder multimorbiden Patienten (Diabetes, Leberzirrhose, fortgeschrittene Arteriosklerose) gedacht werden.

Bei jungen Frauen im gebärfähigen Alter stellen rezidivierende Unterbauchbeschwerden ein häufig auftretendes Problem dar. Die enge anatomische Beziehung des weiblichen inneren Genitales zum Intestinum führt dabei zu besonderen Schwierigkeiten, die Symptome differentialdiagnostisch zuzuordnen.

Bei älteren Menschen sind es neben funktionellen Störungen (Koprostase) auch Tumoren intestinalen oder extraintestinalen Ursprungs, die in die Überlegung einbezogen werden müssen. Sie verursachen neben gelegentlichen Schmerzzuständen häufig eine intermittierende Passagestörung oder eine gastrointestinale Blutung.

Zusätzlich existieren noch einige internistische Krankheitsbilder, die zu rezidivierenden Abdominalbeschwerden führen können, im klinischen Alltag jedoch eher Raritäten (z. B. Kollagenosen, Ogilvie-Syndrom, Purpura Schoenlein-Henoch u. a.) darstellen.

\section{Untersuchungsverfahren}

Im Vordergrund stehen die ausführliche Anamnese und die klinischen Untersuchungen.

Die Anamnese umfaßt eine präzise Erfassung des Schmerzcharakters, der Intensität, Zeitdauer, Lokalisation und Ausstrahlung. Des weiteren ist nach Vorerkrankungen und Voroperationen zu fahnden. Auch die Familien- und Sozialanamnese (Erkrankungen von Kontaktpersonen, familiäre Belastung) kann von richtungsweisender Bedeutung sein.

Die klinische Untersuchung stellt die wichtigste Maßnahme dar und ist entscheidend für die Indikationsstellung zu weiterführenden diagnostischen Prozeduren. Dabei ist die konsiliarische Hinzuziehung anderer Fachgebiete (Gynäkologen, Urologen, Chirurgen, Gastroenterologen) unabdingbar.

Laboruntersuchungen sind in den meisten Fällen nur von nachgeordneter Bedeutung. Wichtig ist die Bestimmung der Entzündungsparameter (Leukozyten, C-reaktives Protein, Blutsenkung). Infektiologische Ursachen werden zusätzlich durch bestimmte serologische Untersuchungen im Blut oder im Stuhl abgeklärt (z. B. Yersiniose, Taeniosen, Salmonellose).

\section{Sonographie}

Unter den apparativen Untersuchungen ist die Sonographie zweifelsfrei das diagnostische Mittel der ersten Wahl. Sie ist nicht invasiv, weist keine Strahlenbelastung auf und kann beliebig oft wiederholt werden. Während sie in der akuten Schmerzsituation in ihrer Aussagekraft durch Darmgasüberlagerung eingeschränkt sein kann, ist der Patient mit chronischem Beschwerdebild in der Regel so vorzubereiten, daß optimale Sichtverhältnisse zu erzielen sind. Die Sonographie erlaubt mit hoher Treffsicherheit die Darstellung morphologischer Veränderungen. Vor allem entzündliche Veränderungen lassen sich aufgrund deutlicher Wandverdickungen, von Ödemen und Exsudatbildung zuverlässig darstellen. So ist die Ultraschalluntersuchung auch für die Verlaufsbeurteilung chronisch entzündlicher Darmerkrankungen das Mittel der Wahl. Das Aufzeigen peritonealer Adhäsionen oder gestörte Darmmotilität ist für den Geübten oft möglich. Bei Nachweis liquider Raumforderungen oder freier Flüssigkeit sind die Punktion dieser Areale unter Sicht und die biochemische und mikrobiologische Analyse des Aspirats möglich. Gleiches gilt für den Ultraschall in der Hand des Urologen und Gynäkologen, hier erweitert durch die transvaginale Sonographie. Zahlreiche Differentialdiagnosen lassen sich durch diese Untersuchung ausschließen oder belegen.

Von herausragender Bedeutung ist jedoch die individuelle Erfahrung des Untersuchers. Eine differenzierte Diagnostik im Bauchraum setzt eine mehrjährige Erfahrung und große Anzahl durchgeführter Ultraschalluntersuchungen voraus.

\section{Röntgendiagnostik}

Im Vergleich zum Ultraschall haben radiologische Verfahren in der Diagnostik von akuten wie auch chronischen Bauchschmerzen eine vergleichsweise geringe Bedeutung und bedürfen einer strengen Indikationsstellung. Dies liegt vor allem in der Strahlenbelastung. Nur wenn die Sonographie Hinweise ergibt, die weiterer Abklärung bedürfen, sollten Röntgenverfahren zur Anwendung kommen. So stellen der Verdacht auf eine chronisch entzündliche Darmerkrankung (Morbus Crohn, Colitis ulcerosa) oder auf einen intraluminalen Tumor gegebenenfalls eine Indikation zur Dünndarmpassage nach Sellink oder zum Kolonkontrasteinlauf dar. Die Computertomogra- 
phie (CT) sollte wenigen Ausnahmesituationen vorbehalten bleiben. So kann sie bei Tumorverdacht, aber auch bei entzündlichen oder abszedierenden Erkrankungen (z. B. Sigmadivertikulitis) indiziert sein. Bei sonographischem Verdacht auf ein Abflußhindernis im Bereich des harnableitenden Systems ist eine Ausscheidungsurographie erforderlich.

Die Magnetresonanztomographie steht für seltene, differenzierte Fragestellungen bei Erkrankungen des Retroperitoneums oder des kleinen Beckens zur Verfügung.

\section{Endoskopie}

Vor allem bei intraluminalen Erkrankungen des Magen-DarmTrakts sind individuell endoskopische Untersuchungsverfahren durchzuführen. So dient die Koloskopie der Abklärung peranaler Blutungen und fraglicher Passagehindernisse. Tumoren, Polypen, entzündliche Stenosen und chronisch entzündliche Darmerkrankungen können hiermit erkannt und mittels bioptischer Sicherung klassifiziert werden.

\section{Laparoskopie}

Die Laparoskopie als diagnostisches Verfahren hat im Rahmen der Verbreitung laparoskopischer Techniken in der operativen Medizin eine neue Bewertung erfahren. Bereits 1902 von Kelling eingeführt, diente dieses Verfahren zunächst fast ausschließlich diagnostischen Zwecken, nämlich der Inspektion und für Biopsien. Therapeutische Eingriffe wurden in den 60er und 70er Jahren vor allem von den Gynäkologen durchgeführt; der diagnostische Einsatz hingegen war aufgrund von Sonographie und CT eher rückläufig. Erst der breite Einsatz der Laparoskopie in der minimalinvasiven Chirurgie ließ das Indikationsspektrum rasch anwachsen. Die damit einhergehende Entwicklung des Instrumentariums erlaubte zudem bei der laparoskopischen Exploration häufig auch eine definitive chirurgische Therapie.

Die Laparoskopie stellt zwar selbst einen operativen Eingriff dar. Da sie aber eine Exploration des gesamten Bauchraums erlaubt, ist ihre Belastung deutlich geringer als die einer Laparotomie mit gleicher Zielsetzung.

Die genaue Inspektion und Exploration des Abdomens erlaubt in vielen Fällen eine eindeutige Diagnose. Falls sich daraus eine konservative Therapie ergibt (z. B. Adnexitis, Ileitis terminalis), wird der Eingriff beendet. Eine operative Therapie kann häufig in gleicher Sitzung laparoskopisch durchgeführt werden (z. B. Adhäsiolyse, Appendektomie) oder erfordert das Umsteigen auf eine offene Operationstechnik. Läßt sich keine eindeutige Ursache für die Schmerzzustände finden, sollte die laparoskopische Appendektomie erfolgen. Die pathohistologische Aufarbeitung zeigt in diesen Fällen häufig trotz unauffälligem makroskopischem Befund mikroskopisch pathologische Veränderungen.

\section{Eigene Patienten}

Im Rahmen einer prospektiven Beobachtungsstudie wurden im Zeitraum von Dezember 1993 bis Juni 1998 die Daten von 100 Pa- tienten erfaßt, die unter der klinischen Diagnose «persistierende oder chronisch rezidivierende rechtsseitige Unterbauchschmerzen» in der Klinik und Poliklinik für Viszeral-, Thorax- und Gefäßchirurgie des Universitätsklinikums Dresden aufgenommen wurden. Anamnese und klinischer Befund, Diagnostik, intraoperativer Befund, durchgeführte Therapie, postoperativer Verlauf (Schmerzskala, Analgetikabedarf) und Histologie wurden anhand standardisierter Erfassungsbögen prospektiv dokumentiert. Nach 3,6 und 12 Monaten erfolgten eine erneute standardisierte Befragung, klinische Untersuchung und sonographische Kontrolle.

Das untersuchte Patientenkollektiv rekrutierte sich aus 81 Frauen und 19 Männern. Das Alter der Frauen betrug im Schnitt 29 Jahre und lag damit deutlich niedriger als bei den Männern mit 38 Jahren.

Die Beschwerdedauer betrug im Mittel 75 Wochen. Die Mindestdauer der Beschwerden war nach Einschlußkriterien mit 14 Tagen festgelegt. 51 Patienten hatten sich bereits mindestens einmal in stationärer Krankenhausbehandlung befunden.

Neben der obligaten Sonographie wurden eine Koloskopie sowie Stuhluntersuchungen auf pathogene Keime durchgeführt. Weitere radiologische und endoskopische Untersuchungen erfolgten im individuellen Fall fakultativ. Alle Patienten wurden diagnostisch laparoskopiert. Die Befunde, die bei der Laparoskopie erhoben wurden, lassen sich in 4 Gruppen unterteilen:

1. Nachweis eines morphologischen Korrelats, welches die Beschwerden unabhängig von der Appendix erklärt ( $\mathrm{n}=14)$.

2. Makroskopisch pathologischer Befund an der Appendix, wie vermehrte Gefäßinjektion, ödematöse Verdickung oder entzündliche Adhäsionen $(\mathrm{n}=50)$.

3. Makroskopisch unauffällige Appendix, aber Adhäsionen im Bereich des Zökalpols, die als Folge abgelaufener Entzündungsschübe gedeutet werden könnten $(\mathrm{n}=21)$.

4. Makroskopisch unauffälliger Abdominalbefund $(\mathrm{n}=15)$.

Während bei den Patienten der Gruppe 1, bei denen ein morphologisches Substrat für die geschilderte Schmerzsymptomatik vorlag, die Appendix belassen wurde, führten wir bei allen anderen $\mathrm{Pa}-$ tienten $(n=86)$ die laparoskopische Appendektomie durch.

Die histologische Untersuchung der Appendix ergab in 82 Fällen $(95,3 \%)$ einen pathologischen Befund. Viermal zeigten sich weder makroskopisch noch mikroskopisch pathologische Veränderungen (zweimal in Gruppe 3, zweimal in Gruppe 4).

Als pathologisch wurden alle Befunde gewertet, die histologische Zeichen der chronischen Appendizitis oder Residuen abgelaufener Entzündungen aufwiesen. Als häufigste histopathologische Veränderung fanden sich

- Fibrose der Mukosa

- follikulär-lymphatische Hyperplasie

- eosinophile Infiltration

- narbige Lumenobliterationen

- katarrhalisch-eitrige Infiltration.

Der postoperative Verlauf war bei $97 \%$ aller Patienten bzw. 96,4\% der appendektomierten Patienten unauffällig. Einmal erfolgte eine Laparotomie am 3. Tag wegen Verdachts auf eine Appendixstumpfinsuffizienz, wobei sich intraoperativ nur auffällig 


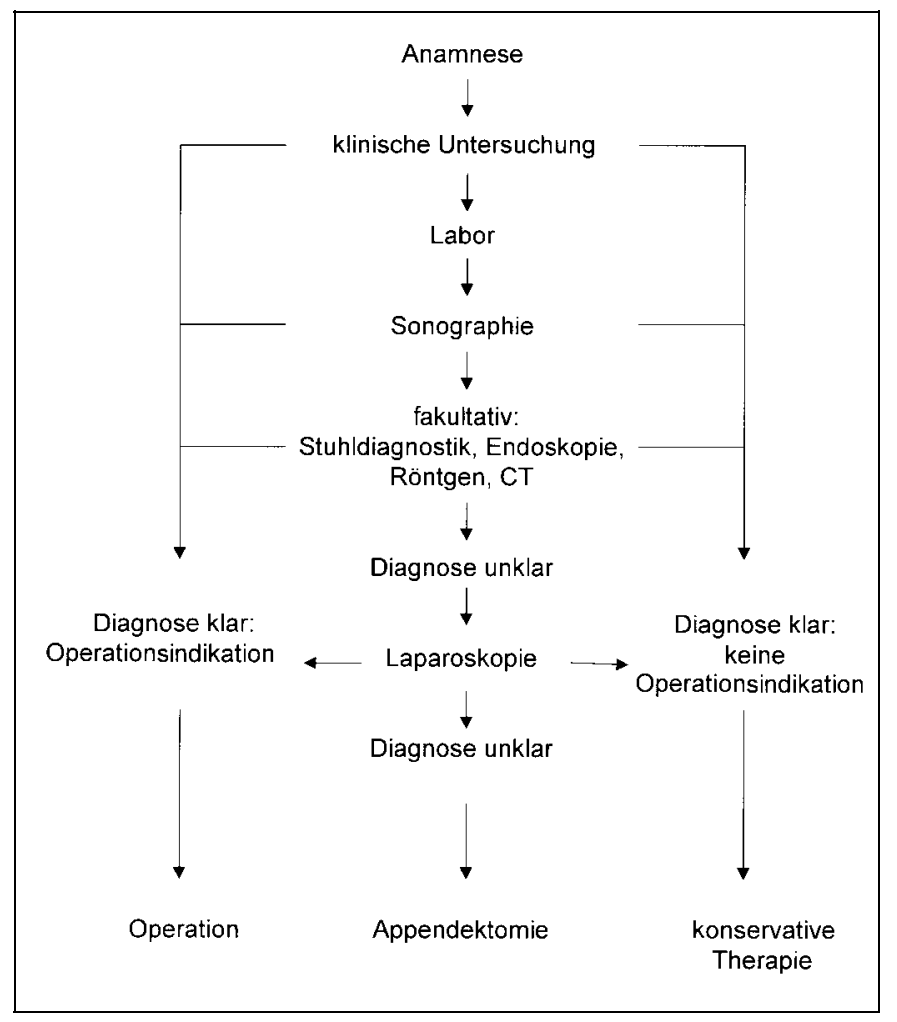

Abb. 1. Diagnostisches Konzept bei chronisch persistierenden oder chronisch rezidivierenden Unterbauchschmerzen.

ausgeprägte Adhäsionen fanden. Zwei Patienten entwickelten eine lokale Wundheilungsstörung im Bereich einer Trokareintrittsstelle. Postoperativ gaben zunächst alle 86 Patienten eine deutliche Beschwerdebesserung an. Nach 3 Monaten waren 80 der appendektomierten Patienten (93\%) schmerzfrei. Sechs Patienten klagten über gleichbleibende oder rezidivierende Schmerzen wie vor der Operation (3 Patienten aus Gruppe 2, 2 Patienten aus Gruppe 3 und 1 Patient aus Gruppe 4). Die Nachuntersuchungen nach 6 und 12 Monaten ergaben bei reduzierter Anzahl an nachbeobachteten Patienten keine weiteren Befundänderungen.

\section{Diskussion}

Chronische oder rezidivierende Unterbauchschmerzen stellen im klinischen Alltag Ärzte und betroffene Patienten häufig vor eine unbefriedigende und frustrierende Situation. Selbst eine ausführliche, oft wiederholt durchgeführte Diagnostik durch verschiedene Fachdisziplinen bringt keine endgültige Klärung. Um die Belastung der Patienten vertretbar zu halten und die diagnostischen Schritte ökonomisch sinnvoll zu gestalten, sollte die Laparoskopie frühzeitig und konsequent in das diagnostische Konzept integriert werden (Abb. 1).

Anamnese und klinischer Befund stehen an erster Stelle, gefolgt von der Ultraschalluntersuchung. Die orientierende Sonographie ist als einzige Maßnahme regelhaft durchzuführen und hat die Aufgabe, neben der eigentlichen Diagnosefindung die Weichen für das weitere Prozedere zu stellen. Alle anderen apparativen oder endoskopischen Untersuchungen bedürfen einer konkreten Fragestellung, die sich aus klinisch-anamnestischen Hinweisen oder aus dem sonographisch unklaren Befund ergibt.

Die Laparoskopie bietet folgende Vorteile:

1. Die diagnostische Aussagekraft ist genauso hoch wie die der Laparotomie, jedoch ist das Operationstrauma deutlich geringer und die perioperative Morbidität niedriger. Dies führt zu kürzeren stationären Liegezeiten und somit geringeren Behandlungskosten. Die Quote an Patienten, bei denen die Laparoskopie eine pathologische Veränderung ergibt, schwankt in der Literatur erheblich. So berichtet Wood von nur 30\% pathologischen Befunden [1]. Andere Autoren hingegen geben zwischen 70 und $82 \%$ pathologische Befunde an [2,3]. Auch die Rate an Ursachen für die Schmerzsymptomatik differiert je nach Definition und Einschlußkriterien. In Studien, in die nur «chronische» Bauchschmerzen aufgenommen wurden, dominieren Verwachsungen [3, 4]. Bei Patienten mit rezidivierenden bzw. «unklaren» Bauchschmerzen hingegen ist die Appendizitis die häufigste Ursache [5, 6].

2. Die Laparoskopie erlaubt neben dem Erstellen einer Diagnose in vielen Fällen auch gleichzeitig eine definitive Therapie [7]. Relativ einfach ist dies bei Patienten, bei denen sich ein eindeutiges morphologisches Korrelat findet. So können neben der Appendizitis Hernien, Ovarialzysten, aber auch Sigmadivertikulitiden laparoskopisch operiert werden [6,8,9]. Die Erfolgsrate bezüglich einer dauerhaften Beschwerdelinderung dieser Patientengruppe ist sehr hoch.

Eine besondere Gruppe stellen Patienten mit peritonealen Adhäsionen mit oder ohne vorausgegangene Operationen dar. Während das morphologische Korrelat der bindegewebigen Verwachsungen laparoskopisch gut behandelbar ist, sind die Langzeitergebnisse unterschiedlich. Übereinstimmend wird jedoch der Vorteil gegenüber der konventionellen Laparotomie zur Adhäsiolyse betont. So ist laparoskopisch eine effektive Adhäsiolyse möglich [10], und die Quote an rezidivierenden Adhäsionen ist gering [11]. Insgesamt kann davon ausgegangen werden, daß in $60-80 \%$ der Patienten nach Adhäsiolyse eine dauerhafte Beschwerdefreiheit zu erzielen ist [12].

Ein Problem stellt die Gruppe von Patienten dar, bei denen sich kein eindeutiges Korrelat für die Beschwerden findet. Übereinstimmend mit den meisten Autoren favorisieren wir in diesem Fall die laparoskopische Appendektomie [6,13,14]. Die Entität einer chronischen oder chronisch rezidivierenden Appendizitis ist weiterhin umstritten. Die Diskussion wurde schon vor der Ära der laparoskopischen Chirurgie geführt und spaltet die Chirurgen in zwei Lager. Während einige Autoren die chronische Appendizitis als eigenständiges Krankheitsbild negieren, wird von anderen der Anteil der primär chronischen oder chronisch rezidivierenden Appendizitis mit $0,5-10 \%$ angegeben. Hinweise auf eine chronisch entzündliche Affektion der Appendix können peritoneale Adhäsionen zur Appendix oder zum Zökalpol sein. Aber auch bei makroskopisch völlig blander Appendix kann die pathohistologische Untersuchung häufig mikroskopische Veränderungen aufzeigen [14-16]. Diese reichen von frisch exazerbierten Schüben, akuter 
katarrhalischer bis eitriger Entzündung über lymphozytäre oder eosinophile Infiltrationen bis hin zur Fibrose der Submukosa oder narbigen Lumenobliteration.

Das wichtigste Zielkriterium für die Beantwortung der Frage der Existenz einer chronischen oder chronisch rezidivierenden Appendizitis ist die Remission der Schmerzen nach Appendektomie. Der Erfolg scheint hierbei die Indikation zur Appendektomie zu bestätigen. So wird die Rate an beschwerdefreien Patienten nach Appendektomie wegen chronischer Appendizitis mit 85-100\% angegeben $[16,17]$.

\section{Zusammenfassung}

Bei chronischen und chronisch rezidivierenden Unterbauchschmerzen stellt die Laparoskopie ein sicheres und komplikationsarmes Verfahren zur Diagnostik dar. In einem hohen Prozentsatz erlaubt die visuelle Exploration des Bauchraums nicht nur eine Diagnose, sondern in gleicher Sitzung auch eine definitive Therapie. Findet sich kein morphologisches Korrelat, sollte bei diesen Patienten die laparoskopische Appendektomie durchgeführt werden.

\section{Literatur}

1 Wood RAB, Cuschieri A: Laparoscopy for chronic abdominal pain. Br J Surg 1997;60:900-902.

2 Klingensmith ME, Soybel DI, Brooks DC: Laparoscopy for chronic abdominal pain. Surg Endosc 1996;10:1085-1087.

3 Nagy AG, James D: Diagnostic laparoscopy. Am J Surg 1989;157:490-493.

4 Lavonius M, Gullichsen R, Laine S, Ovaska J: Laparoscopy for chronic abdominal pain. Surg Laparosc Endosc 1999;9:42-44.

5 Linos DA, Vlitaki M, Peppas G, Tsakayannis D: Laparoscopy in the context of lower abdominal pain in young women. J Laparoendosc Adv Surg Tech A 1999;1:39-43.

6 Miller K, Mayer E, Moritz E: The role of laparoscopy in chronic and recurrent abdominal pain. Am J Surg 1996;172:353-356.

7 Salky BA, Edye MB: The role of laparoscopy in the diagnosis and treatment of abdominal pain syndromes. Surg Endosc 1998;12:911-914.
8 Bruch HP, Schiedeck T: Der unklare Unterbauchschmerz - Stellenwert der Laparoskopie. Chirurg 1997;68:12-16.

9 Lippert V, Zaage J, Pilz F: Diagnostische Laparoskopie und laparoskopische Appendektomie im Diagnostik- und Therapiekonzept des unklaren Unterbauchschmerzes. Zentralbl Chir 1998;123: 46-49.

10 Nezhat CR, Nezhat FR, Metzger DA, Luciano AA: Adhesion reformation after reproductive surgery by videolaparoscopy. Fertil Steril 1990; 53:1008-1010.

11 Operative Laparoscopy Study Group: Postoperative adhesion development after operative laparoscopy: Evaluation at early second look procedures. Fertil Steril 1991;55:700-704.

12 Mueller MD, Tschudi J, Herrmann U, Klaiber C: An evaluation of laparoscopic adhesiolysis in patients with chronic abdominal pain. Surg Endosc 1995;9:802-804.
13 Seidman JD, Andersen DK, Ulrich S: Recurrent abdominal pain due to chronic appendiceal disease. South Med J 1991;84:913-916.

14 Mattei P, Sola JE, Yeo CJ: Chronic and recurrent appendicitis are uncommon entities often misdiagnosed. J Am Coll Surg 1994;178:385-389.

15 Wehrmann U, Stoelben E, Schilling B, Nagel M, Saeger HD: Die diagnostische Laparoskopie Stellenwert in der Diagnostik und Therapie des chronisch rezidivierenden Unterbauchschmerzes. Langenbecks Arch Chir 1995;2:510-512.

16 Crabbe MM, Norwood SH, Robertson HD, Silva JS: Recurrent and chronic appendicitis. Surg Gynecol Obstet 1986;163:11-13.

17 Fayez JA, Toy NJ, Flanagan TM: The appendix as the cause of chronic lower abdominal pain. Am J Obstet Gynecol 1995;72:122-123. 